\title{
The AAVSO and its Variable Star Databank on Pulsating Stars
}

\author{
Janet A. Mattei
}

AAVSO, 25 Birch Street,Cambridge, MA 02138, USA

\begin{abstract}
The American Association of Variable Stars Observers is the world's largest organization of variable star observers. Its services to the astronomical community and database on pulsating variables are described.
\end{abstract}

\section{Organization and services}

The American Association of Variable Star Observers (AAVSO) coordinates, collects, evaluates and archives variable star observations made largely by amateur astronomers worldwide; publishes variable star data and disseminates them to researchers and educators around the world. Over 7.8 million observations have been incorporated into the AAVSO International Database since its founding in 1911. The AAVSO receives over 300,000 mostly visual observations yearly. Observations for inclusion in the database are standardized, homogeneous and subject to quality control checks to ensure the highest level of reliability.

The AAVSO receives over 200 requests each year from astronomers for its data and services to help schedule observing runs using ground-based and satellite telescopes; to provide simultaneous optical coverage of observing targets and immediate notification of their activity during particular satellite observing programs; to correlate multi-wavelength data; and to carry out collaborative research to analyze the long-term behaviour of variable stars.

\section{Data on pulsating stars}

There are over 2100 pulsating variable stars of Mira (1217), semiregular (586), Cepheid (179), RV Tauri (36), R Coronae Borealis (35), Symbiotic (34), and S Doradus (13) types in the AAVSO observing program. For many hundreds of stars, the AAVSO has almost continuous visual observations going back many decades, using consistent sets of comparison stars. Sample light curves from AAVSO International Database from 1963 to mid-1994 are shown in Figure 1. AAVSO observations and published information on maxima and minima dates and magnitudes of long period variables going back to 1900 have been used to correlate light curve parameters; to study period and pulsation mode changes; to search for chaotic behaviour; and to investigate stellar evolution. For AAVSO data and services, please contact the Director at the address above or by e-mail at aavso@cfa.harvard.edu. 
I gratefully acknowledge AAVSO and IAU grants that made it possible for me to attend this well-organized, interesting and enjoyable meeting.
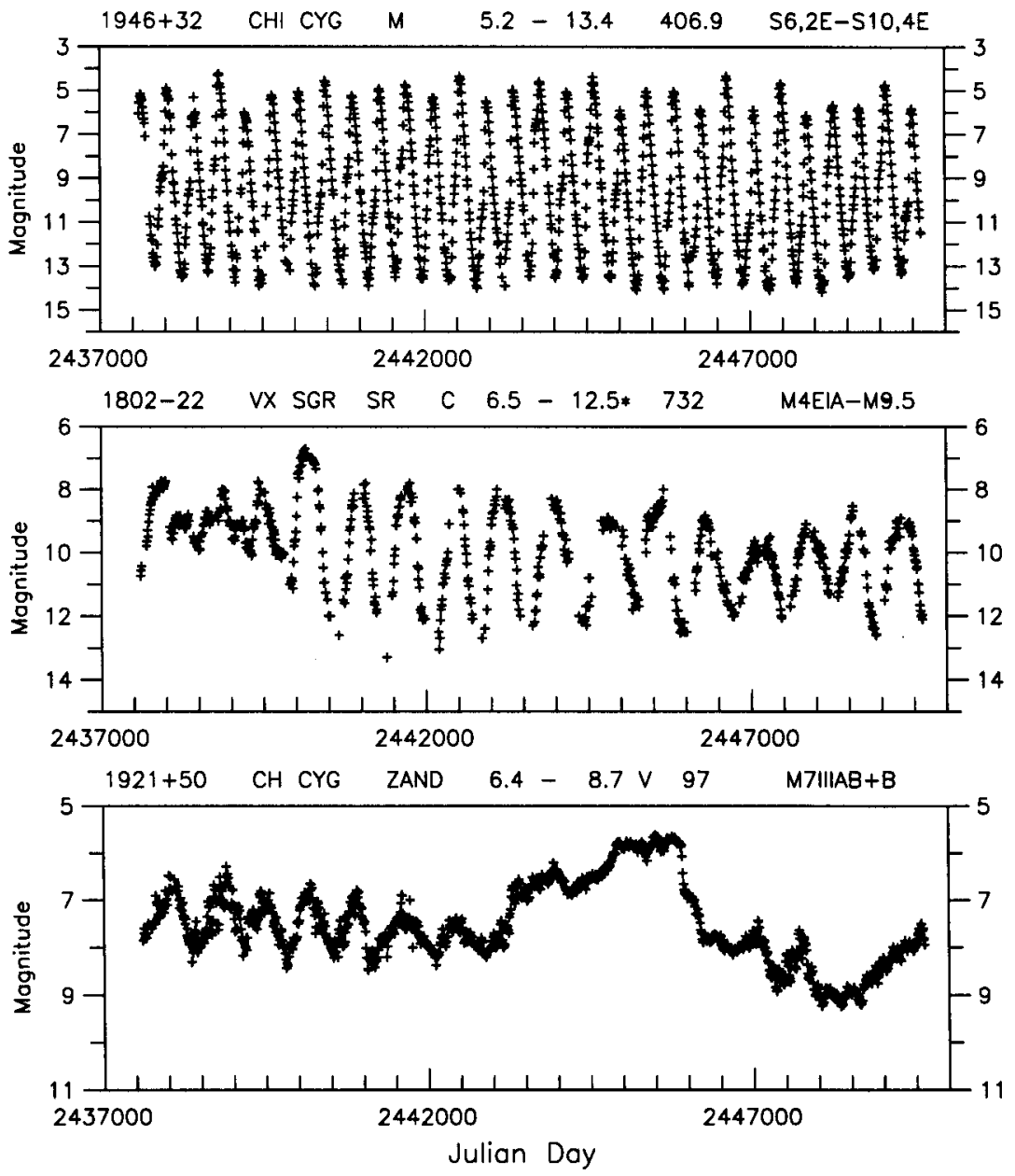

Figure 1. Sample light curves from the AAVSO International Database of (top to bottom) $\chi \mathrm{Cyg}, \mathrm{VX} \mathrm{Sgr,} \mathrm{and} \mathrm{CH} \mathrm{Cyg.} \mathrm{Each}$ cross represents 10-day means of observations. 